\title{
The Effect of the Vertex Angles of Wedged Indenters on Deformation during Nanoindentation
}

\author{
Xiaowen $\mathrm{Hu}$ (D) and Yushan $\mathrm{Ni}^{*}$ \\ Department of Aeronautics and Astronautics, Fudan University, Shanghai 200433, China; \\ xwhu16@fudan.edu.cn \\ * Correspondence: niyushan@fudan.edu.cn \\ Academic Editors: Ronald W. Armstrong, Stephen M. Walley and Wayne L. Elban \\ Received: 30 September 2017; Accepted: 12 December 2017; Published: 14 December 2017
}

\begin{abstract}
In order to study the effect of the angle of wedged indenters during nanoindentation, indenters with half vertex angles of $60^{\circ}, 70^{\circ}$ and $80^{\circ}$ are used for the simulations of nanoindentation on FCC aluminum (Al) bulk material by the multiscale quasicontinuum method (QC). The load-displacement responses, the strain energy-displacement responses, and hardness of Al bulk material are obtained. Besides, atomic configurations for each loading situation are presented. We analyze the drop points in the load-displacement responses, which correspond to the changes of microstructure in the bulk material. From the atom images, the generation of partial dislocations as well as the nucleation and the emission of perfect dislocations have been observed with wedged indenters of half vertex angles of $60^{\circ}$ and $70^{\circ}$, but not $80^{\circ}$. The stacking faults move

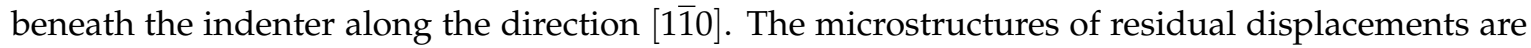
also discussed. In addition, hardness of the Al bulk material is different in simulations with wedged indenters of half vertex angles of $60^{\circ}$ and $70^{\circ}$, and critical hardness in the simulation with the $70^{\circ}$ indenter is bigger than that with the $60^{\circ}$ indenter. The size effect of hardness in plastic wedged nanoindentation is observed. There are fewer abrupt drops in the strain energy-displacement response than in the load-displacement response, and the abrupt drops in strain energy-displacement response reflect the nucleation of perfect dislocations or extended dislocations rather than partial dislocations. The wedged indenter with half vertex angle of $70^{\circ}$ is recommended for investigating dislocations during nanoindentation.
\end{abstract}

Keywords: nanoindentation; multiscale simulation; wedged indenter; dislocation nucleation and emission; size effect of hardness during indentation

\section{Introduction}

With the increase of application of micro-electronic devices, nanoindentation has been widely used as a standard method to obtain mechanical properties such as nanohardness and elastic modulus of materials [1-4]. Both experimental and numerical research have been implemented to observe the microstructures near the indenter tip in recent years. In terms of experimental studies, for instance, C. A. Schuh et al. $[5,6]$ used a Triboindenter to investigate the dislocation nucleation and incipient plasticity during high-temperature nanoindentation on $\mathrm{Pt}$ single crystal, and obtained mechanical properties of standard fused silica at temperature up to $405^{\circ} \mathrm{C}$. D. Ge et al. [7] employed a three-sided pyramidal Berkovich tip to investigate the size effect in the nanoindentation of silicon. Sandra Korte et al. [8] implemented a high-temperature nanoindentation of $\mathrm{Au}$ in vacuum, and obtained values of properties like Young moduli, yield and flow stresses. However, experimental nanoindentation tests are sensitive to the environment and instrument [5-8]. Numerical simulation becomes an effective method to study the basic mechanical behaviors in nanoindentation. 
When it comes to numerical methods, the finite element (FE) method is often employed to simulate nanoindentation. For example, J. A. Knapp et al. [9] developed procedures of nanoindentation based on finite-element modeling, accurately calculated some mechanical properties and compared them with experiments. Kaushal K Jha et al. [10] studied the physical meaning of the total and elastic energy constants in elasto-plastic indentations by Finite-element method, and discussed their applications in the evaluation of nanomechanical quantities such as the indenter tip radius and the nominal hardness. However, it is hard to validly simulate the changes of microstructures near the indenter tip, such as dislocations and twinnings. Moreover, some drop points of load-displacement curve are hard to obtain. Molecular dynamics (MD) is another widely employed method to study the microscopic mechanisms. Many researchers studied nanoindentation with this method. Landman et al. [11], for the first time, simulated nanocontact problems using Ni tips with MD method. Yen-Hung Lin et al. [12] simulated the nanoindentation on monocrystalline silicon with both spherical and Berkovich indenters, and discussed the nanoindentation-induced deformation and the phase transformation during the process. Jiang et al. [13] simulated the nanoindentations on a binary metallic glass under various strain rates, and validated that the serration is not directly dependent on the resultant shear-banding spatiality. Although the MD method can provide a lot of details of micro-deformation, such like dislocation nucleation and emission, it requires lots of calculations that will limit the simulation both on the time scale and the model size. The quasi-continuum (QC) method is one of the multiscale methods which allows relatively large model to simulate the mechanical behaviors in nanoscale. Many researchers have studied micro behaviors of nanomaterials with this method. Huaibao Lu et al. $[14,15]$ investigated the effect of surface step on nanoindentation in various orientations by the QC method. Aibin Zhu et al. [16] simulated the nanoindentation on single crystal cooper by QC method, and discussed the effect of radius of sphere indenters on nanohardness. Mei and Ni [17] studied the anisotropic behavior during nano-adhesive contact by the QC method, which proves the importance of crystal orientation of micro-devices. Fanlin Zeng et al. [18] investigated the titled flat-ended nanoindentation with different titled angles by the QC method, and simulation results agreed well with analytical ones.

Wedge indentation is an important process for determining the mechanical properties of materials, and angles of conical and wedged indenters affect the measurement of mechanical properties greatly. D. S. Dugdale [19] conducted the wedge indentation experiments with three different cold-worked metals, and found that the measured hardnesses was independent of size when the metal was larger than a certain size. K. Eswar Prasad et al. [20] investigated the role of the angle of conical indenters on the plastic deformation during nanoindentation by using the FE method, and compared the results with experimental data. Fan-lin Zeng et al. [21] simulated the wedged nanoindentation on nickel by using indenters with different vertex angles, and studied the dislocation emission beneath the indenter tip. However, the unloading nanoindentation processes with different angles of wedged indenters have not been studied, and the influence of the angle of wedged indenters on the hardness of materials has not been discussed yet. In this paper, we simulate the loading and unloading nanoindentation processes on Al bulk material by using wedged indenters with different vertex angles by QC method. Load-displacement responses and energy-displacement responses were obtained. Hardness of materials in different wedged nanoindentations were calculated too. In particular, the mechanism of dislocation emission will be discussed, and the residual dislocation during the unloading process will also be investigated.

\section{Methodology and Simulation Model of Nanoindentation}

The QC method is an effective multiscale approach to simulate the mechanical behaviors of crystalline materials, which couples the continuum and atomic simulation. It is established that discrete atomic descriptions are only necessary at highly deformed regions and vicinity of defects or interfaces, while the linear elastic continuum method is employed in other regions. In the QC method, there are two kinds of representative atoms called local atoms and non-local atoms. The local atoms 
gather the deformation behavior of atoms that go through a nearly homogeneous deformation whose energies are computed from the local deformation gradients based on Cauchy-Born continuum rule. On the other hand, the non-local atoms are treated by Ercolessi-Adams Al potential [22] to describe the atomic movement in the area where heterogeneous deformations occur. The QC method runs through molecular static energy minimization of all atoms over the atomic (non-local) domain and the finite element (local) domain. Meanwhile, the QC method automatically reduces the degrees of freedom by implementing an automatic adaption scheme with no atomistic detail loss in the core region. The sizes of atomistic region and finite element region are constantly updated and expands during the simulation, and the selection of representative atoms and their local versus nonlocal status is automatically carried out by a formulation using a certain criteria [23] In this case, the atomistic zone of the model is big enough to capture plastic deformation and nucleation and emission of dislocations. The dislocations in the material will always be located in the atomistic region. In addition, the models used in the QC method are pseudo-2D models [24]. This means that although the analysis is performed in a two-dimestional coordinate system, the displacements in the z-direction are allowed and all atomistic calculations are three-dimensional. Within this setting, only dislocations with line directions perpendicular to the plane of analysis can be nucleated and the displacement fields were constrained to have no variation in the out-of-plane z-direction. These constraints appear to be compatible with the two-dimensional nature of the indenter. This is a form of generalized plane strain, which could effectively simplify our simulation. More details of QC method are discussed in $[23,24]$.

The nanoindentation model of this work is illustrated in Figure 1. Wedged indenters with different semi-angles $\left(\alpha=60^{\circ}, 70^{\circ}\right.$ and $\left.80^{\circ}\right)$ were pressed into aluminum (Al) single crystal bulk material. The model is $100 \mathrm{~nm}$ thick, $200 \mathrm{~nm}$ wide, and infinite in the out-of-plane direction with periodic boundary conditions. In addition, the size of the initial atomistic region is $10 \mathrm{~nm}$ wide and $2 \mathrm{~nm}$ thick. The model is much larger than most of models used in MD nanoindentation simulations. The crystal orientations of the model along $x-, y-$, and $z$-axis are chosen to be [111], [110] and [1 12$]$, respectively, which could promote the emissions of Shockley partial dislocations and deformation twinning [25]. A fixed boundary condition is applied to the bottom surface, and free boundary conditions are applied to the top, left and right surfaces. The indenter was settled on the middle of the top surface, and the indenter was gradually pushed into the film along $y$-direction with an increment of $0.01 \mathrm{~nm}$ per step. The interactions between the atoms are described by Ercolessi-Adams Al potential [22], and the elastic moduli predicted by this potential are $C_{11}=117.74 \mathrm{GPa}, C_{12}=62.02 \mathrm{GPa}$ and $C_{44}=36.76 \mathrm{GPa}$. The effective values of shear modulus $G=33.14 \mathrm{GPa}$ and Poisson's ratio $v=0.319$ are calculated by a Voigt average [24]:

$$
\begin{gathered}
G=\frac{1}{5}\left(C_{11}-C_{12}+3 C_{44}\right) \\
v=\frac{1}{2}\left[\frac{C_{11}+4 C_{12}-2 C_{44}}{2 C_{11}+3 C_{12}+C_{44}}\right]
\end{gathered}
$$

Thus lattice constant $a=0.4032 \mathrm{~nm}$. Finally, indenters penetrated $1.2 \mathrm{~nm}$ deep into the material.

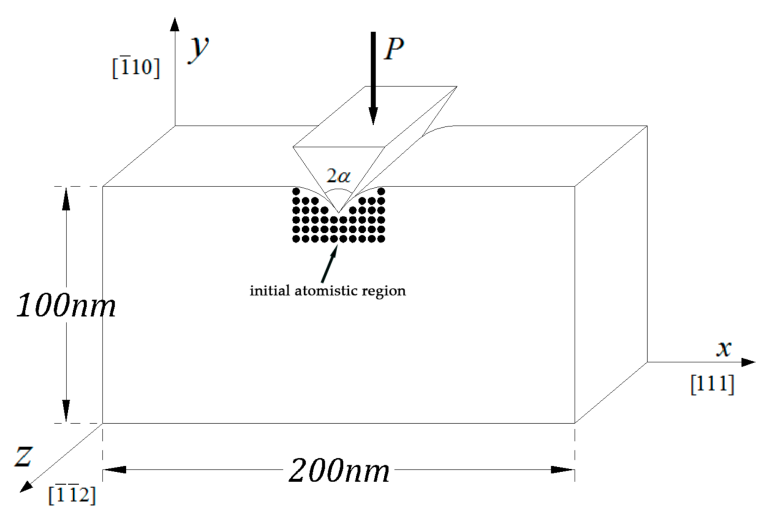

Figure 1. Schematic representation of wedged nanoindentation. 


\section{Results and Discussion}

\subsection{Load-Displacement Responses}

\subsubsection{Load-Displacement Responses during Different Loading Processes}

We present the load-displacement responses of nanoindentation loading processes using wedged indenters with half vertex angles ( $\alpha$ in Figure 1 ) of $60^{\circ}, 70^{\circ}$ and $80^{\circ}$ in Figure 2 . Loads are calculated in Newton per meter length in the $\mathrm{z}$ direction of the $\mathrm{y}$ directional resultant force on the indenters. At the very beginning of indentations, the curves of three different loading processes are almost the same. This is because the contact parts of three indenters are almost the same at the beginning, which is called the tip radius effect [26]. When the contact width exceeds the tip width, the curves become different. It is clear that load goes up with the increase of indentation depth at the beginning of three different indentations. These initial deformation stages are regarded as the elastic stages. As indenters are pressed deeply, the pop-in effect is investigated in nanoindentation with both half vertex angles of $60^{\circ}$ and $70^{\circ}$. As it is shown, the load drops suddenly at a depth of $4.7 \AA$ (Point $\mathrm{A}_{1}$ ) with a critical load $\boldsymbol{P}_{c r 60}=3.22 \mathrm{~N} / \mathrm{m}$ during nanoindentation with the indenter of $60^{\circ}$. As the indenter proceeds more deeply, the load continues to increase with several drops. Three different drop points that happen at $4.7 \AA, 7.7 \AA$ and $10.7 \AA\left(\mathrm{A}_{1}, \mathrm{~B}_{1}\right.$ and $\left.\mathrm{C}_{1}\right)$ are marked, while three abrupt drops occur at $5.6 \AA, 8.1 \AA$ and $10.4 \AA\left(\mathrm{A}_{2}, \mathrm{~B}_{2}\right.$, and $\left.\mathrm{C}_{2}\right)$ during nanoindentation using the wedged indenter of $70^{\circ}$, as shown in Figure 2. The critical load during nanoindentation with the $70^{\circ}$ indenter is $\boldsymbol{P}_{\boldsymbol{c r}} \mathbf{7 0}=3.99 \mathrm{~N} / \mathrm{m}$. However, in nanoindentation with the $80^{\circ}$ indenter, the load smoothly increases with the increase of indentation depth and no abrupt drop occurs. In addition, the load increases with the increase of angle of wedged indenter when the indentation depth is the same. The elastic stage of nanoindentation with the indenter of $70^{\circ}$ continues longer than that with the indenter of $60^{\circ}$. It is known that the drop points in the load-displacement response indicate the dislocation nucleation and emission, which will be discussed below.

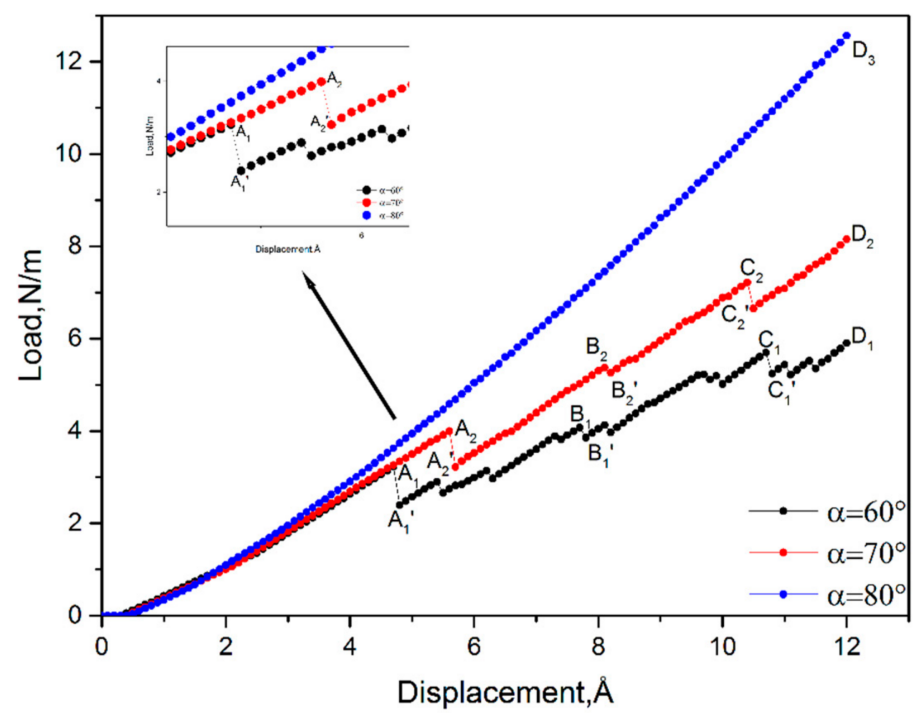

Figure 2. Load-displacement responses for wedged nanoindentation on Al single crystal by using indenters with different vertex angles.

\subsubsection{Load-Displacement Responses during Different Unloading Processes}

As we discussed above, several drop points are observed in loading processes. It is essential to confirm whether the plastic deformation happens at these points. The indenter with half vertex angle of $60^{\circ}$ is retracted from $\mathrm{A}_{1}{ }^{\prime}$ and $\mathrm{D}_{1}$, as shown in Figure 3, while the indenter of $70^{\circ}$ is retracted from $\mathrm{A}_{2}{ }^{\prime}, \mathrm{B}_{2}{ }^{\prime}$ and $\mathrm{C}_{2}{ }^{\prime}$, as shown in Figure 4 . 


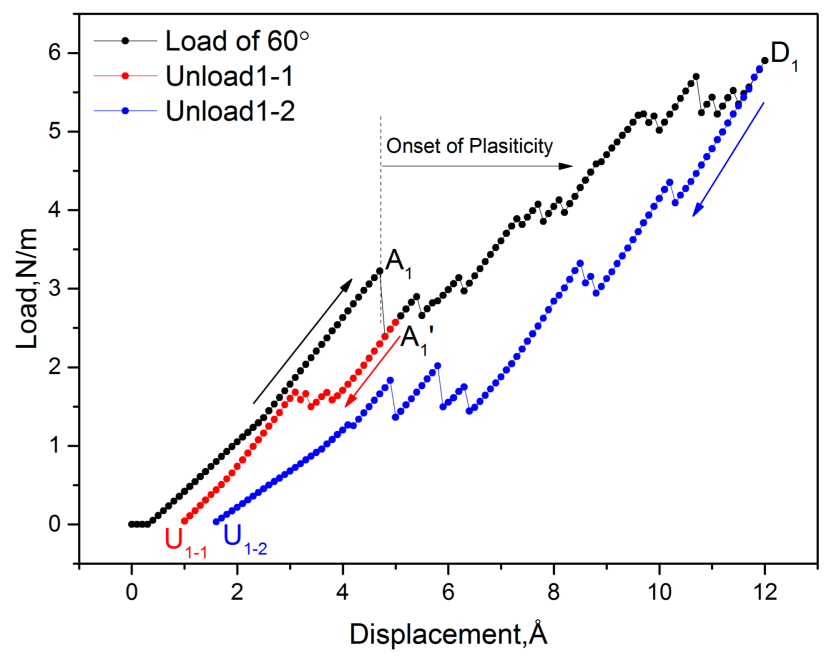

Figure 3. Load-displacement responses during unloading processes using the wedged indenter with half vertex angle of $60^{\circ}$.

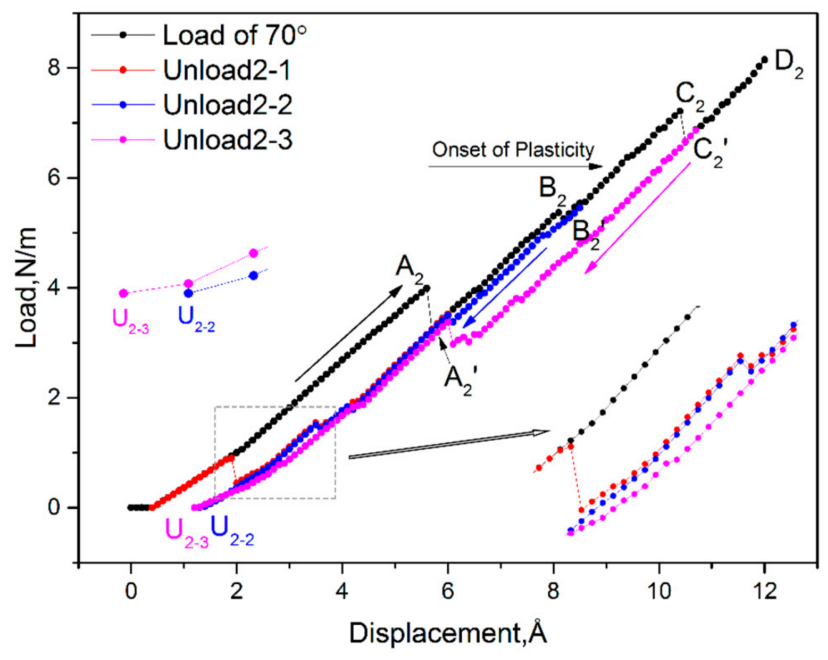

Figure 4. Load-displacement responses during unloading processes using the wedged indenter with half vertex angle of $70^{\circ}$.

In Figure 3, we perform unloading processes with the indenter of half vertex angle of $60^{\circ}$ at $A_{1}{ }^{\prime}$ and $\mathrm{D}_{1}$ as "Unload1-1" and "Unload1-2", respectively. During "Unload1-1", the load decreases as the indentation depth decreases. The value of load fluctuates when indentation depth declines from $3.8 \AA$ to $3.1 \AA$, and then the load linearly decreases to zero with a residual displacement $\mathrm{U}_{1-1}=0.9 \AA$. The residual displacement of unloading process is the indentation depth when the load on the indenter decreases to zero. During "Unload1-2" process, two abrupt jumps of load occur at $10.2 \AA$ and $8.5 \AA$. After the displacement fluctuates from $6.4 \AA$ to $5.0 \AA$, the load smoothly drops to zero with a residual displacement $\mathrm{U}_{1-2}=1.5 \AA$. These residual displacements prove that irreversible plastic deformation occurs in indentation loading process after point $\mathrm{A}_{1}{ }^{\prime}$.

In Figure 4, retractions from $\mathrm{A}_{2}{ }^{\prime}, \mathrm{B}_{2}{ }^{\prime}$ and $\mathrm{C}_{2}{ }^{\prime}$ were exhibited as "Unload2-1", "Unload2-2", and "Unload2-3", respectively. As is shown, the load from $\mathrm{A}_{2}{ }^{\prime}$ decreases with the decrease of indentation depth. When indentation depth falls to $1.9 \AA$, the load abruptly jumps up to the curve of loading process and then decreases back to the origin with no residual displacement. This means the deformation happened at point $\mathrm{A}_{2}$ is recoverable. During "Unload2-2" process, the load decreases from $\mathrm{B}_{2}$ ' and no obviously abrupt jump occurs, which is different from "Unload2-1" process. Finally, the load decreases 
to zero with a residual displacement $U_{2-2}=1.3 \AA$. As for "Unload2-3", the load decreases gradually where an abrupt jump happens at $6.0 \AA$. Then, the load decreases to zero with a residual displacement $\mathrm{U}_{2-3}=1.2 \AA$, which is almost the same as $\mathrm{U}_{2-2}$. The residual displacements are attributed to the changes of microscopic structures of the material during unloading processes and will be discussed below. The pop-out effect that happens during the unloading process also agrees with many experiments, and it is considered to be relevant to the atomic rearrangement of the bulk material [7,27].

The load-displacement response of unloading process with the indenter of $80^{\circ}$ is also obtained. The curve of unloading process completely coincides with the curve of loading process. This suggests that the loading process with the indenter of $80^{\circ}$ is an elastic process. On the other hand, the load-displacement responses of three different nanoindentations show good linearity at the initial stage of unloading processes, which also agrees with many experimental results [28,29].

\subsection{Atom Configurations of Dislocation Nucleation and Emission}

3.2.1. Atomic Configurations during Nanoindentation Using the Indenter with Half Vertex Angle of $60^{\circ}$

In order to understand the mechanism of the drop points in the load-displacement responses, the atomic details beneath the indenter tip need to be observed. The Von Mises effective strain distribution and atomic configurations of abrupt drops during nanoindentation using the wedged indenter with half vertex angle of $60^{\circ}$ are shown in Figure $5 \mathrm{a}-\mathrm{d}$ correspond to points $\mathrm{A}_{1}{ }^{\prime}, \mathrm{B}_{1}{ }^{\prime}, \mathrm{C}_{1}{ }^{\prime}$ and $\mathrm{D}_{1}$ in Figure 2, respectively). As we mentioned in Section 2, the line directions of dislocations in QC method are all perpendicular to the plane of analysis. By standard, we use " $\perp$ " symbol to label the dislocations in atom configurations [30]. The projection of Burgers vector is parallel to the bottom line of " $\perp$ ", and the direction of this projection vector is from left to right. The Burgers vectors of the dislocations are determined by the directions of their projection vectors and the Thompson tetrahedron in FCC metals [31]. We also plot the slip direction of dislocations in the configurations. The discussion about dislocation nucleation and emission goes as follow.

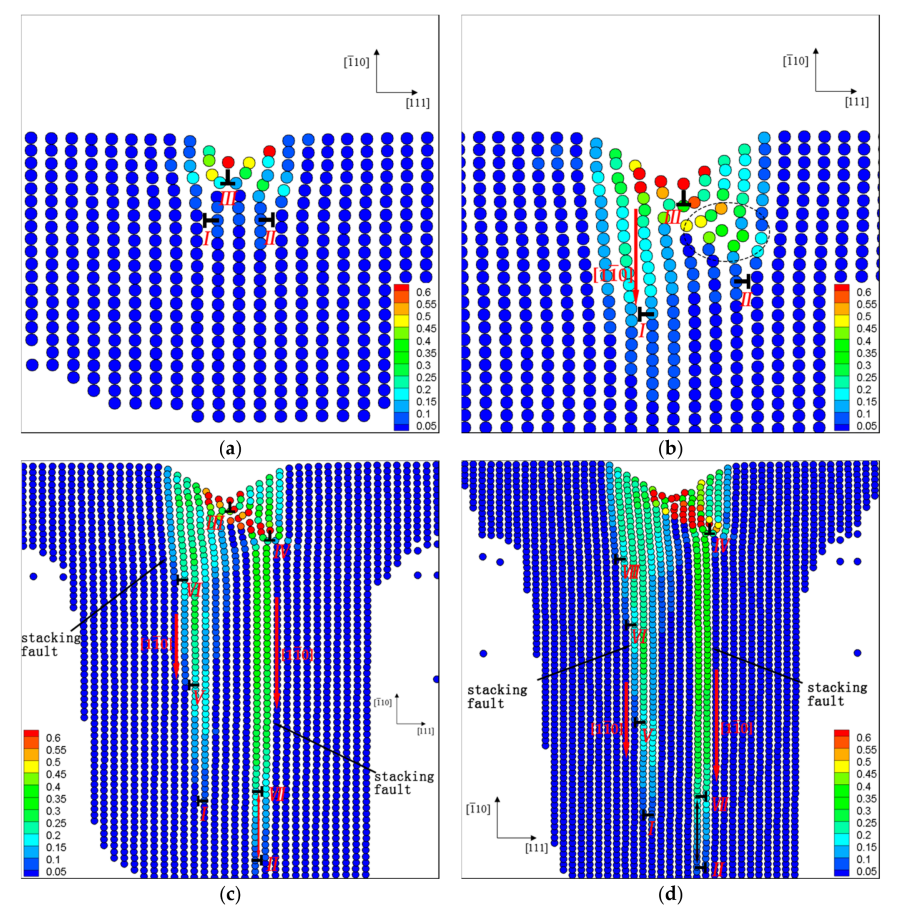

Figure 5. Strain distribution and dislocations beneath the wedged indenter with vertex angle of $60^{\circ}$ during loading nanoindentation process: (a) $\mathrm{h}=4.8 \AA$, (b) $\mathrm{h}=7.8 \AA$, (c) $\mathrm{h}=10.8 \AA$, (d) $\mathrm{h}=12.0 \AA$. 
During loading process of $60^{\circ}$, when the indenter is pressed into material $4.6 \AA$, a dislocation dipole (a pair of Shockley partials, $1 / 6[2 \overline{1} \overline{1}]$ and $1 / 6[\overline{2} 11]$ ) nucleates beneath the indenter shoulder at point I and II (Figure 5a). This is the first emergence of geometrically necessary dislocations [32]. In addition, a perfect dislocation $1 / 2[110]$ emerges at point III. This dislocation nucleation corresponds to the drop point $\mathrm{A}_{1}$ ' (in Figure 2), which means that the nucleation of dislocations will reduce the force on the indenter and release the stress concentration on the zone around the indenter tip. When the indentation depth comes to $7.7 \AA$ (point $\mathrm{B}_{1}{ }^{\prime}$ in Figure 2), the dislocation dipole is dissociated, as shown in Figure 5b. Shockley partial II moves below, and a lot of disordered atoms stack above the dislocation

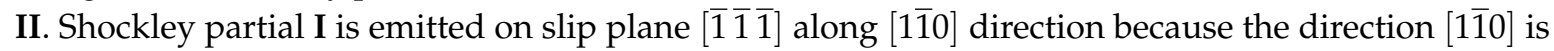
a favorable slip direction for FCC metals [24]. When indentation depth continues to increase to $10.8 \AA$ (point $\mathrm{C}_{1}{ }^{\prime}$ ), a 1/2[110] perfect dislocation emerges at point IV, as shown in Figure $5 \mathrm{c}$. The formation of this dislocation is due to the stacking of disordered atoms in Figure 5b. The Shockley partials I and II continue to slide on [ $\overline{1} \overline{1} \overline{1}]$ plane along [110] direction. Three new Shockley partials appear, among which two $(1 / 6[\overline{2} 11]$, marked as $\mathbf{V}$ and $\mathbf{V I})$ appear beside the slip planes, which are generated by the emission of Shockley partial I, and the third Shockley partial VII $(1 / 6[1 \overline{2} 1])$ appears in the stacking fault $18.9 \AA$ above the Shockley partial II. In addition, the Shockley partial II and VII could be considered as dissociated from a perfect dislocation 1/2[110]. These two dislocations and stacking fault between them (as the line connected in Figure 5c) constitute an extended dislocation. In Figure 5d, the perfect dislocation III disappears, and other dislocations continue moving down. Then a new Shockley partial VIII emerges. As it can be seen from Figure $5 c$,d, the distance between the stacking faults is four atoms' width. Thus, the width of the zone where partial dislocations move is eight atoms' width in the loading process with the indenter of $60^{\circ}$.

To confirm the residual displacements after retractions, the atom configurations of material after unloading processes by using the indenter with half vertex angle of $60^{\circ}$ at $\mathrm{A}_{1}{ }^{\prime}$ and $\mathrm{D}_{1}$ are plotted in Figure 6 ( $(a)$ and $(b)$ correspond to $U_{1-1}$ and $U_{1-2}$ in Figure 3, respectively).

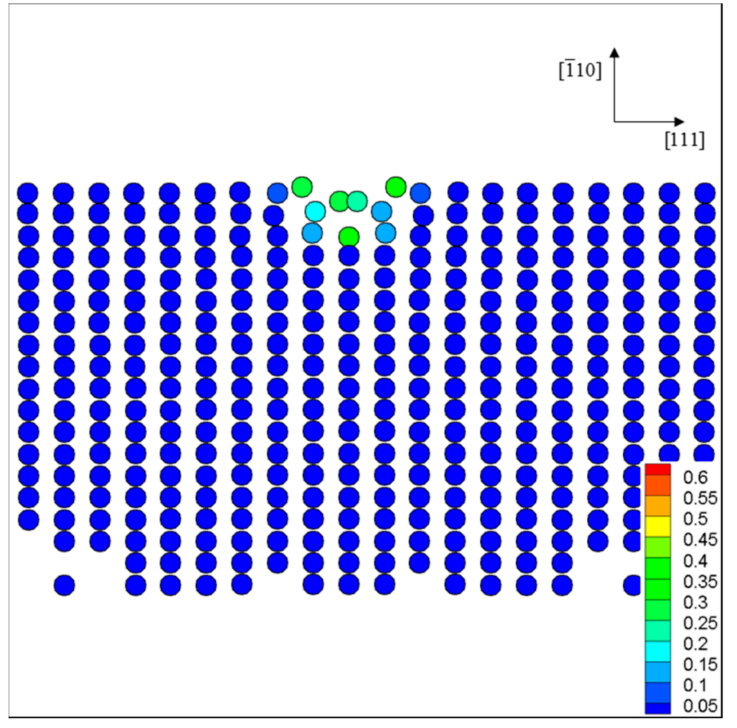

(a)

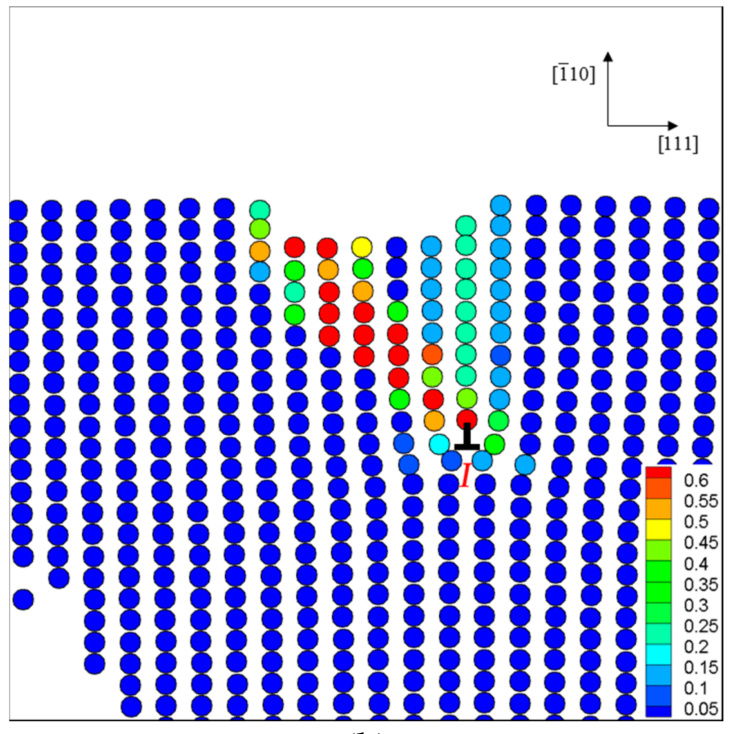

(b)

Figure 6. Strain distribution and atom configurations of Al bulk material under the wedged indenter with half vertex angle of $60^{\circ}$ after retractions at $A_{1}{ }^{\prime}$ and $D_{1}^{\prime}$ : (a) $U_{1-1} ;\left(\right.$ b) $U_{1-2}$.

After retraction process at $\mathrm{A}_{1}{ }^{\prime}$, several disordered atoms stack at the surface as shown in Figure $6 \mathrm{a}$. The dislocations have disappeared, which could be explained by the following model [24]. The forces on the dislocations include the Peach-Koehler force $\left(\boldsymbol{F}_{P K}\right)$ due to the indenter stress field pushing the dislocation into the bulk material, and the image force $\boldsymbol{F}_{\boldsymbol{I}}$ pulling the dislocation back to the 
surface. These two forces and Peierls stress $\sigma_{p}$ maintain a balance when dislocation is stationary. It is described as

$$
F_{P K}+F_{I}=b \sigma_{p}
$$

where $\boldsymbol{b}$ is module of Burgers vector. When the indenter is pulled back, the Peach-Koehler force applied on the dislocation decreases, the image force would dominate and the dislocation would return to the surface and disappear.

In Figure $6 \mathrm{~b}$, the atomic configurations of bulk material after retraction from $\mathrm{D}_{1}{ }^{\prime}$ are presented. Compared to the atom configuration at $\mathrm{D}_{1}$ in loading process, only one perfect dislocation $\mathbf{I}$ is trapped in the bulk material, and other dislocations have moved back to the surface and disappeared. Because the module of Burgers vector of perfect dislocation is bigger than that in partial dislocation, the Peierls stress $\sigma_{p}$ applied on this perfect dislocation dominates. Thus, the image force from the surface cannot pull it back, and the dislocation remains in the material. In this sense, perfect dislocation is a stable microstructure in residual displacement.

3.2.2. Atomic Configurations during Nanoindentation Using the Indenter with Half Vertex Angle of $70^{\circ}$

In Figure 2, three different abrupt drops of load are investigated during nanoindentation by using the indenter with half vertex angle of $70^{\circ}$. The atom configurations of these points are extracted to observe the microstructure changes at these moments, as shown in Figure 7 ((a), (b), (c) correspond to points $\mathrm{A}_{2}{ }^{\prime}, \mathrm{B}_{2}{ }^{\prime}, \mathrm{C}_{2}{ }^{\prime}$ in Figure 2, respectively). In Figure $7 \mathrm{a}$, the atomic configuration of the zone beneath the wedged indenter with half vertex angle of $70^{\circ}$ at point $A_{2}{ }^{\prime}$ is presented. A dislocation dipole (two Shockley partials I and II) emerges beneath the indenter shoulder, and a $1 / 2[110]$ perfect dislocation nucleates at point III. Both are also observed in the loading process with the indenter of $60^{\circ}$. As shown in Figure 8b, when displacement comes to $8.2 \AA$ (point $\mathrm{B}_{2}{ }^{\prime}$ ), dislocation I slides on slip plane $[\overline{1} \overline{1} \overline{1}]$ along $[1 \overline{1} 0]$ direction, while former dislocation II in Figure 7a disappears. When the indenter is pressed into the bulk material $10.5 \AA$ (point $C_{2}{ }^{\prime}$ ), as shown in Figure $7 c$, a new Shockley partial II $(1 / 6[2 \overline{1} \overline{1}])$ is emitted, and two stacking fault zones emerge beneath the indenter shoulder. The dislocation I continues moving down, and two new Shockley partials nucleate on new slip planes $[\overline{1} \overline{1} \overline{1}]$ beside the slip plane where dislocation $\mathbf{I}$ locates. This also happens in the loading process with the indenter of $60^{\circ}$. Another Shockley partial VI (1/6[121]) nucleates $17.5 \AA$ above dislocation II. Dislocations II and VI and the stacking fault between them constitute an extended dislocation. In addition, the distance between two stacking faults in loading process with the indenter of $70^{\circ}$ is seven atoms' width. The width of zone that partial dislocations move is 11-atoms width, which is wider than that in nanoindentation with the indenter of $60^{\circ}$.

The atomic configurations of the bulk material after unloading processes with the indenter of $70^{\circ}$ retracted from $B_{2}{ }^{\prime}$ and $C_{2}{ }^{\prime}$ are plotted in Figure $8\left((a)\right.$ and $(b)$ correspond to $U_{2-2}$ and $U_{2-3}$ in Figure 4, respectively). As shown in Figure $8 \mathrm{a}$, when retraction from $\mathrm{B}_{2}{ }^{\prime}$ ends, one perfect dislocation remains at surface. This means that unrecoverable plastic deformation has happened at $\mathrm{B}_{2}{ }^{\prime}$ during loading process. In Figure $8 b$, three perfect dislocations remain in the film when retraction from $C_{2}$ ' ends, which are arrayed as a line parallel to the surface. The pattern they are arranged leads to the fact that the residual displacement of these three dislocations is almost the same as that of one dislocation $\left(U_{2-2}\right.$ and $\left.U_{2-3}\right)$, as we mentioned in Section 3.1.2. From the discussion of atomic configurations of residual displacements, it is clear that perfect dislocations rather than partial dislocations will remain in the material when retraction process ends. Additionally, more dislocations in bulk material with the indenter of $70^{\circ}$ will remain than those with the indenter of $60^{\circ}$. 


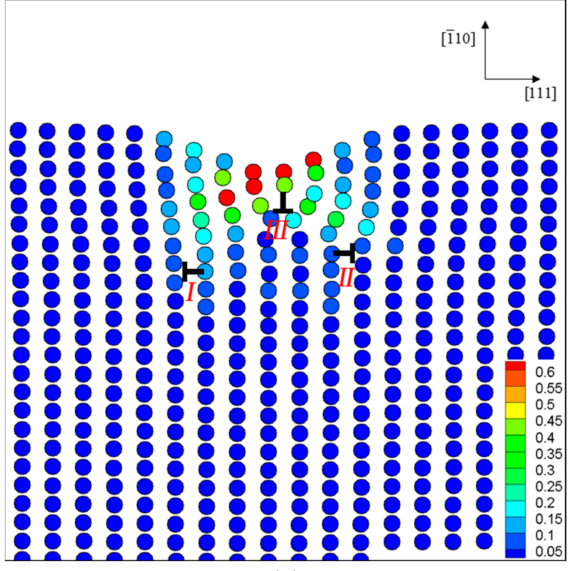

(a)

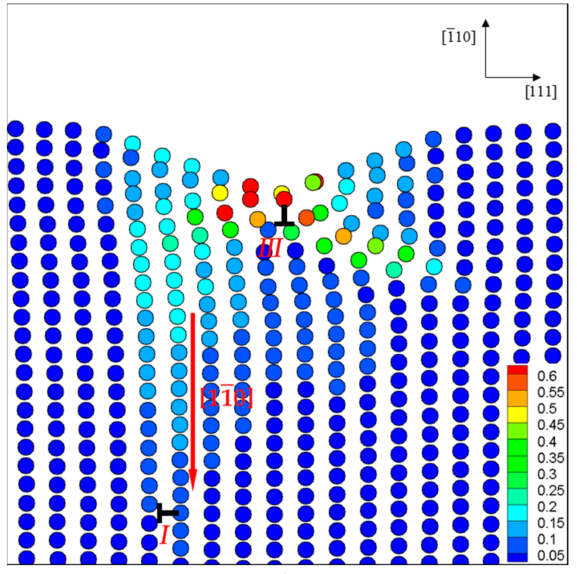

(b)

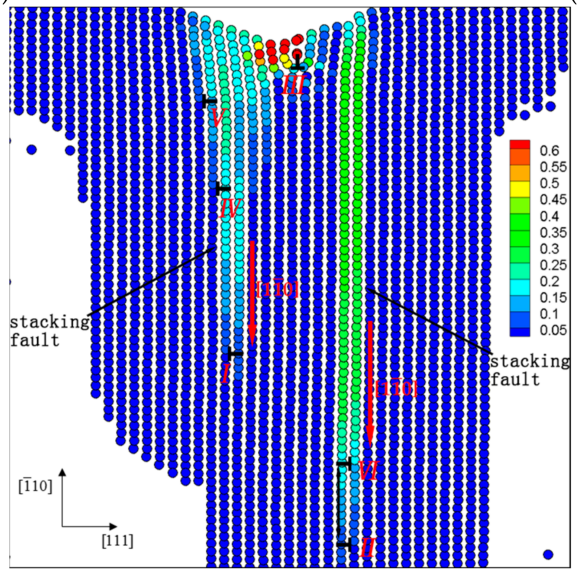

(c)

Figure 7. Strain distribution and dislocations beneath the wedged indenter with half vertex angle of $70^{\circ}$ during loading nanoindentation process: (a) $h=5.7 \AA$, (b) $h=8.2 \AA$, (c) $h=10.5 \AA$.

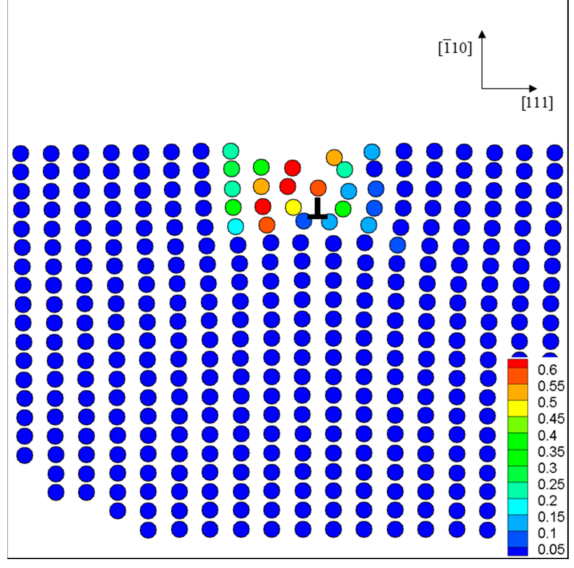

(a)

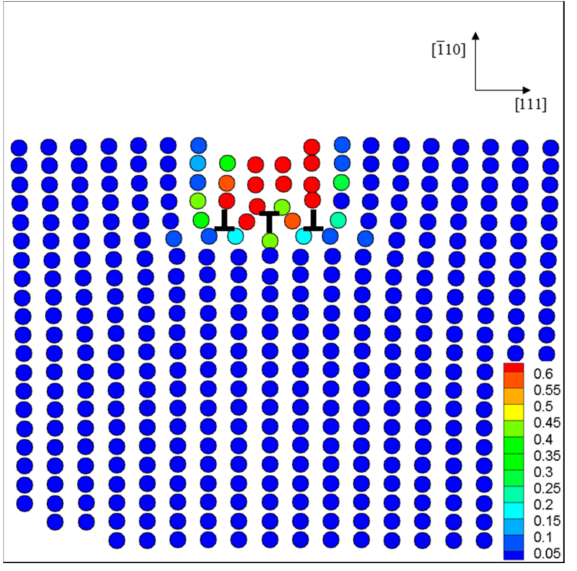

(b)

Figure 8. Strain distribution and atom configurations of $\mathrm{Al}$ bulk material under the wedged indenter with half vertex angle of $70^{\circ}$ after retractions at $\mathrm{B}_{2}{ }^{\prime}$ and $\mathrm{C}_{2}{ }^{\prime}$ : (a) $\mathrm{U}_{2-2} ;(\mathbf{b}) \mathrm{U}_{2-3}$.

As the analysis of atomic configurations is shown above, the mechanisms of dislocation nucleation and emission in wedged nanoindentations with indenters of $60^{\circ}$ and $70^{\circ}$ are similar. The dislocation zones in nanoindentation with both indenters of $60^{\circ}$ and $70^{\circ}$ extend deeply inside the bulk material, but the width would not exceed the contact width, which is defined as the width of the segment 
where indenter is in contact with the material. This phenomenon also agrees with many indentation experiments $[33,34]$. The width of the zone where partial dislocations move in the loading process with the indenter of $70^{\circ}$ is wider than that in loading process of $60^{\circ}$, and the amount of dislocations that remain in the material after retraction with the indenter of $70^{\circ}$ is larger than that with the indenter of $60^{\circ}$. Thus, the indenter with half vertex angle of $70^{\circ}$ is a better choice for investigating dislocation nucleation and emission and for understanding residual displacement during wedged nanoindentation.

\subsection{Contact Hardness Responses}

Hardness of solids is a measure of resistance that a solid matter shows to various kinds of local permanent deformation [35], and hardness during indentation is defined as [1,36]:

$$
H=\frac{P}{A_{p}}
$$

where $P$ is the load, and $A_{p}$ is the projected area. In our simulation, the projected area is contact width multiplied by the length of the indenter in z-direction. The hardness can be calculated by dividing load $P$ by contact width. The projected area is a discrete parameter that increases periodically with the increase of indentation depth, because it is related to the number of atoms which are in contact with the indenter. Thus, there is a critical hardness during nanoindentation. The critical hardness of wedged nanoindentations with different half vertex angles of $60^{\circ}, 70^{\circ}$ are listed in Table 1 . Results show that critical hardness of wedge nanoindentation with half vertex angle of $70^{\circ}$ is larger than that with half vertex angle of $60^{\circ}$. This also agrees with the result of conical indenter studied by Alhafez et al [37].

Table 1. Critical hardness of wedged nanoindentations

\begin{tabular}{cc}
\hline Half Vertex Angle of Wedged Indenter & Critical Hardness (GPa) \\
\hline$\alpha=60^{\circ}$ & 7.018 \\
$\alpha=70^{\circ}$ & 7.295 \\
\hline
\end{tabular}

The size effect of contact hardness with contact depth is a typical characteristic of nanoindentation. As discuss above, the contact width and depth in our simulations are discrete parameters. More than one value of hardness is obtained at the same contact depth. Thus, the calculated hardness $H$ is a range for each, and this range is shown as an error bar. The calculated results of hardness are plotted in Figure 9. As shown, calculating results of hardness in loading processes with both indenters of $60^{\circ}$ and $70^{\circ}$ show dependence on the contact depth. As the contact depth increases, the indentation hardness has a tendency to decrease. This phenomenon also agrees with many other researchers' work [38-42].

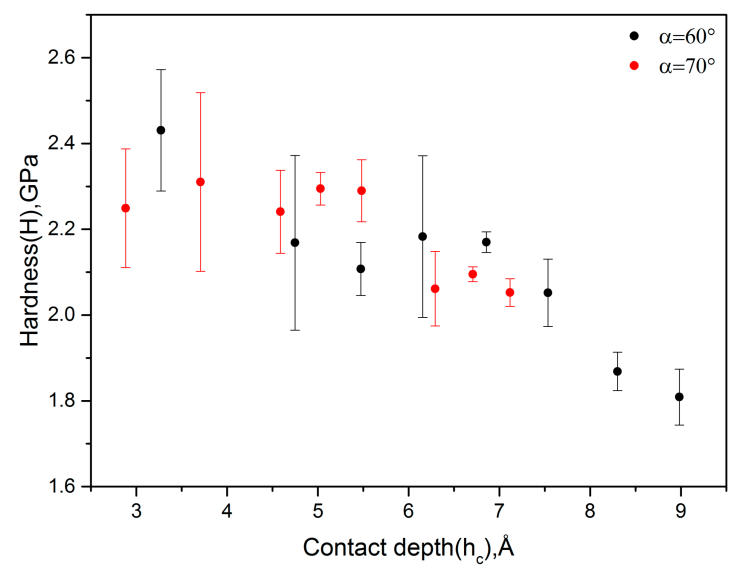

Figure 9. Correlation between hardness and contact depth in wedged nanoindentation with different half vertex angles of $60^{\circ}$ and $70^{\circ}$. 


\subsection{Strain Energy-Displacement Responses}

As we mentioned before, the QC method runs through the minimization of energy of the system. The strain energy in the QC method is the total energy of all atoms over the atomic (non-local) domain and the finite element (local) domain [23]. The strain energy versus displacement responses for wedged nanoindentations with half vertex angles of $60^{\circ}, 70^{\circ}$ and $80^{\circ}$ were obtained, as shown in Figure 10. The strain energy increases with the increase of indentation depth, and two obvious drops occur at point $A_{1}{ }^{\prime}$ and $C_{1}{ }^{\prime}$ in response to indentation with the indenter of $60^{\circ}$, where strain energy drops down $0.593 \mathrm{eV}$ and $0.924 \mathrm{eV}$, respectively. The drop points of strain energy in Figure 10 correspond to the drop points of load in Figure 2, so we use same letters. Two drops occur in strain energy-displacement response when half vertex angle of the indenter is $70^{\circ}$, in which strain energy drops down $0.850 \mathrm{eV}$ and $2.016 \mathrm{eV}$ at point $\mathrm{A}_{2}{ }^{\prime}$ and $\mathrm{C}_{2}{ }^{\prime}$. It is obvious that there are fewer drop points in strain energy-displacement response than in the load-displacement response. According to the atomic configurations shown in Section 3.2, these drop points in strain-energy responses all correspond to the nucleation of perfect dislocations or emergence of extended dislocations rather than partial dislocations. However, in load-displacement response, the drop points correspond to the nucleation and emission of all kinds of dislocations, including partial dislocations. Thus, in wedged nanoindentation, the abrupt drops in strain energy-displacement response only reflect the nucleation of perfect dislocations or extended dislocations.

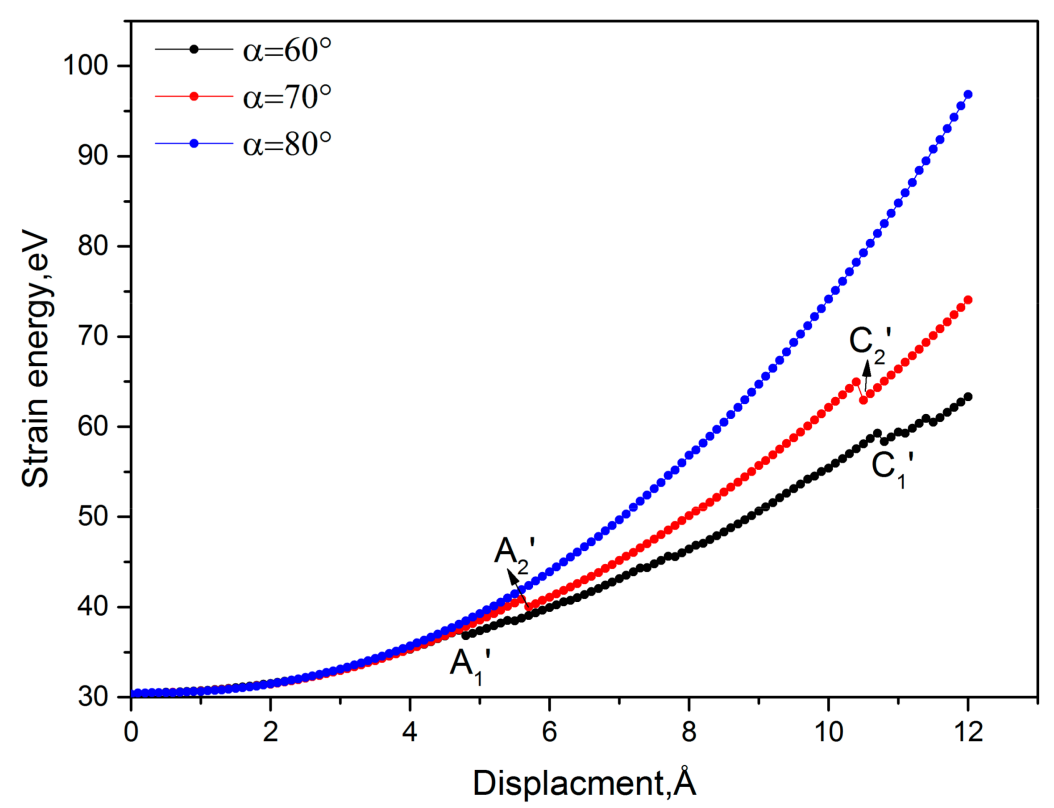

Figure 10. Strain energy-displacement responses of wedged nanoindentations with half vertex angles of $60^{\circ}, 70^{\circ}$ and $80^{\circ}$.

\section{Conclusions}

The effect of the vertex angle of wedged indenters on deformation of $\mathrm{Al}$ single crystal bulk material during nanoindentation was investigated by the QC method. Wedged indenters with half vertex angles of $60^{\circ}, 70^{\circ}$ and $80^{\circ}$ are used to simulate the loading and unloading nanoindentation processes. Load-displacement responses of loading and unloading processes, strain energy-displacement responses, hardness, and dislocation nucleation and emission for three kinds of indenters are analyzed. Results show that some abrupt drops occur in the load-displacement responses of nanoindentation with indenters of $60^{\circ}$ and $70^{\circ}$, and these points correspond to the dislocation nucleation and emission. No abrupt drop occurs during the loading process of the indenter of $80^{\circ}$, and the unloading curve overlaps the loading curve, suggesting that in this simulation with that included angle the indentation 
is purely an elastic process. From the analysis of atomic configurations, the mechanisms of dislocation nucleation and emission in wedged nanoindentation with indenters of $60^{\circ}$ and $70^{\circ}$ are discussed, and the movements of partial dislocations are investigated. Results show that only perfect dislocations will remain when the retraction of indenter ends. Although residual displacements are almost the same, the microstructures of them can be different. It is clear that the dislocation zone beneath the indenter extends deeply, but the width of plastic zone does not exceed the contact width. The width of zone where partial dislocations move during indentation with the indenter of $70^{\circ}$ is wider than that in indentation with the indenter of $60^{\circ}$. In addition, the size effect of hardness in plastic wedged nanoindentations with indenters of $60^{\circ}$ and $70^{\circ}$ is observed. There are fewer abrupt drops in the strain energy-displacement response than in the load-displacement response, and the abrupt drops in strain energy-displacement response in wedged nanoindentation only reflect the nucleation of perfect dislocations or extended dislocations rather than partial dislocations. In summary, the wedged indenter with half vertex angle of $70^{\circ}$ is recommended for investigating the dislocations and for understanding residual displacements during elastic-plastic nanoindentation.

Acknowledgments: This work is supported by the National Natural Science Foundation of China (Grant No. 11572090).

Author Contributions: Xiaowen Hu conducted the simulation, analyzed the data and wrote the paper. Yushan Ni conceived and supervised the whole work, and also revised the paper.

Conflicts of Interest: The authors declare no conflict of interest.

\section{References}

1. Oliver, W.C.; Pharr, G.M. An improved technique for determining hardness and elastic modulus using load and displacement sensing indentation experiments. J. Mater. Res. 1992, 7, 1564-1583. [CrossRef]

2. Pethicai, J.B.; Hutchings, R.; Oliver, W.C. Hardness measurement at penetration depths as small as $20 \mathrm{~nm}$. Philos. Mag. A 2006, 48, 593-606. [CrossRef]

3. Tao, S.; Li, D.Y. Tribological, mechanical and electrochemical properties of nanocrystalline copper deposits produced by pulse electrodeposition. Nanotechnology 2006, 17, 65-78. [CrossRef]

4. Krauss, A.R.; Auciello, O.; Gruen, D.M.; Jayatissa, A.; Sumant, A.; Tucek, J.; Mancini, D.C.; Moldovan, N.; Erdemir, A.; Ersoy, D.; et al. Ultrananocrystalline diamond thin films for MEMS and moving mechanical assembly devices. Diam. Relat. Mater. 2001, 10, 1952-1961. [CrossRef]

5. Schuh, C.A.; Mason, J.K.; Lund, A.C. Quantitative insight into dislocation nucleation from high-temperature nanoindentation experiments. Nat. Mater. 2005, 4, 617-621. [CrossRef] [PubMed]

6. Schuh, C.A.; Packard, C.E.; Lund, A.C. Nanoindentation and contact-mode imaging at high temperatures. J. Mater. Res. 2006, 21, 725-736. [CrossRef]

7. Ge, D.; Minor, A.M.; Stach, E.A.; Morris, J.W. Size effects in the nanoindentation of silicon at ambient temperature. Philos. Mag. 2006, 86, 4069-4080. [CrossRef]

8. Korte, S.; Stearn, R.J.; Wheeler, J.M.; Clegg, W.J. High temperature microcompression and nanoindentation in vacuum. J. Mater. Res. 2012, 27, 167-176. [CrossRef]

9. Knapp, J.A.; Follstaedt, D.M.; Myers, S.M.; Barbour, J.C.; Friedmann, T.A. Finite-element modeling of nanoindentation. J. Appl. Phys. 1999, 85, 1460-1474. [CrossRef]

10. Jha, K.K.; Zhang, S.; Suksawang, N.; Wang, T.L.; Agarwal, A. Work-of-indentation as a means to characterize indenter geometry and load-displacement response of a material. J. Phys. D Appl. Phys. 2013, 46, 415501. [CrossRef]

11. Landman, U.; Luedtke, W.D.; Burnham, N.A.; Colton, R.J. Atomistic mechanisms and dynamics of adhesion, nanoindentation, and fracture. Science 1990, 248, 454-461. [CrossRef] [PubMed]

12. Lin, Y.H.; Jian, S.R.; Lai, Y.S.; Yang, P.F. Molecular dynamics simulation of nanoindentation-induced mechanical deformation and phase transformation in monocrystalline silicon. Nanoscale Res. Lett. 2008, 3, 71-75. [CrossRef]

13. Jiang, S.Y.; Jiang, M.Q.; Dai, L.H.; Yao, Y.G. Atomistic origin of rate-dependent serrated plastic flow in metallic glasses. Nanoscale Res. Lett. 2008, 3, 524-529. [CrossRef] [PubMed] 
14. Lu, H.B.; Ni, Y.S. Effect of surface step on nanoindentation of thin films by multiscale analysis. Thin Solid Films 2012, 520, 4934-4940. [CrossRef]

15. Lu, H.B.; Ni, Y.S.; Mei, J.F.; Li, J.W.; Wang, H.S. Anisotropic plastic deformation beneath surface step during nanoindentation of FCC Al by multiscale analysis. Comput. Mater. Sci. 2012, 58, 192-200. [CrossRef]

16. Zhu, A.B.; He, D.Y.; He, R.J.; Zou, C. Nanoindentation simulation on single crystal copper by quasi-continuum method. Mater. Sci. Eng. A-Struct. 2016, 674, 76-81. [CrossRef]

17. Mei, J.F.; Ni, Y.S. The study of anisotropic behavior of nano-adhesive contact by multiscale simulation. Thin Solid Films 2014, 566, 45-53. [CrossRef]

18. Zeng, F.L.; Zhao, B.; Sun, Y. Multiscale simulation of incipient plasticity and dislocation nucleation on nickel film during tilted flat-ended nanoindentation. Acta Mech. Solida Sin. 2015, 28, 484-496. [CrossRef]

19. Dugdale, D.S. Wedge indentation experiments with cold-worked metals. J. Mech. Phys. Solids 1953, 2, 14-26. [CrossRef]

20. Prasad, K.E.; Chollacoop, N.; Ramamurty, U. Role of indenter angle on the plastic deformation underneath a sharp indenter and on representative strains: An experimental and numerical study. Acta Mater. 2011, 59, 4343-4355. [CrossRef]

21. Zeng, F.L.; Sun, Y.; Liu, Y.Z.; Zhou, Y. Multiscale simulations of wedged nanoindentation on nickel. Comput. Mater. Sci. 2012, 62, 47-54. [CrossRef]

22. Ercolessi, F.; Adams, J.B. Interatomic potentials from first-principles calculations: The force-matching method. EPL-Europhys. Lett. 1994, 26, 583-588. [CrossRef]

23. Shenoy, V.B.; Miller, R.; Tadmor, E.B.; Rodney, D.; Phillips, R.; Ortiz, M. An adaptive finite element approach to atomic-scale mechanics-The quasicontinuum method. J. Mech. Phys. Solids 1999, 47, 611-642. [CrossRef]

24. Tadmor, E.B.; Miller, R.; Phillips, R.; Ortiz, M. Nanoindentation and incipient plasticity. J. Mater. Res. 1999, 14, 2233-2250. [CrossRef]

25. Li, J.W.; Ni, Y.S.; Wang, H.S.; Mei, J.F. Effects of crystalline anisotropy and indenter size on nanoindentation by multiscale simulation. Nanoscale Res. Lett. 2010, 5, 420-432. [CrossRef] [PubMed]

26. Qu, S.; Huang, Y.; Nix, W.D.; Jiang, H.; Zhang, F.; Hwang, K.C. Indenter tip radius effect on the Nix-Gao relation in micro- and nanoindentation hardness experiments. J. Mater. Res. 2004, 19, 3423-3434. [CrossRef]

27. Cross, G.; Schirmeisen, A.; Stalder, A.; Grutter, P.; Tschudy, M.; Durig, U. Adhesion interaction between atomically defined tip and sample. Phys. Rev. Lett. 1998, 80, 4685-4688. [CrossRef]

28. Rubio, G.; Agrait, N.; Vieira, S. Atomic-sized metallic contacts: Mechanical properties and electronic transport. Phys. Rev. Lett. 1996, 76, 2302-2305. [CrossRef] [PubMed]

29. Stalder, A.; Durig, U. Study of yielding mechanics in nanometer-sized Au contacts. Appl. Phys. Lett. 1996, 68, 637-639. [CrossRef]

30. Hull, D.; Bacon, D.J. Chapter 1-Defects in crystals. In Introduction to Dislocations, 5th ed.; Butterworth-Heinemann: Oxford, UK, 2011; pp. 1-20.

31. Hull, D.; Bacon, D.J. Chapter 5-Dislocations in face-centered cubic metals. In Introduction to Dislocations, 5th ed.; Butterworth-Heinemann: Oxford, UK, 2011; pp. 85-107.

32. Ashby, M.F. The deformation of plastically non-homogeneous materials. Philos. Mag. 1970, $21,399-424$. [CrossRef]

33. Chaudhri, M.M. Subsurface strain distribution around Vickers hardness indentations in annealed polycrystalline copper. Acta Mater. 1998, 46, 3047-3056. [CrossRef]

34. Srikant, G.; Chollacoop, N.; Ramamurty, U. Plastic strain distribution underneath a Vickers Indenter: Role of yield strength and work hardening exponent. Acta Mater. 2006, 54, 5171-5178. [CrossRef]

35. Tabor, D. The hardness of solids. Rev. Phys. Technol. 1970, 1, 145. [CrossRef]

36. Oliver, W.C.; Pharr, G.M. Measurement of hardness and elastic modulus by instrumented indentation: Advances in understanding and refinements to methodology. J. Mater. Res. 2004, 19, 3-20. [CrossRef]

37. Alhafez, I.A.; Brodyanski, A.; Kopnarski, M.; Urbassek, H.M. Influence of tip geometry on nanoscratching. Tribol. Lett. 2017, 65, 1-13. [CrossRef]

38. Nix, W.D.; Gao, H. Indentation size effects in crystalline materials: A law for strain gradient plasticity. J. Mech. Phys. Solids 1997, 46, 411-425. [CrossRef]

39. Manika, I.; Maniks, J. Size effects in micro- and nanoscale indentation. Acta Mater. 2006, 54, $2049-2056$. [CrossRef] 
40. Durst, K.; Goken, M.; Pharr, G.M. Indentation size effect in spherical and pyramidal indentations. J. Phys. D Appl. Phys. 2008, 41, 074005. [CrossRef]

41. Pharr, G.M.; Herbert, E.G.; Gao, Y.F. The indentation size effect: A critical examination of experimental observations and mechanistic interpretations. In Annual Review Of Materials Research; Clarke, D.R., Ruhle, M., Zok, F., Eds.; Annual Reviews: Palo Alto, CA, USA, 2010; Volume 40, pp. 271-292.

42. Ma, L.; Morris, D.J.; Jennerjohn, S.L.; Bahr, D.F.; Levine, L.E. The role of probe shape on the initiation of metal plasticity in nanoindentation. Acta Mater. 2012, 60, 4729-4739. [CrossRef] 